\title{
Managing arthralgia in a postmenopausal woman taking an aromatase inhibitor for hormone- sensitive early breast cancer: a case study
}

This article was published in the following Dove Press journal:

Cancer Management and Research

28 March 2012

Number of times this article has been viewed

\author{
Jane Bryce' \\ Martina Bauer ${ }^{2}$ \\ Peyman Hadji² \\ 'National Cancer Institute, Naples, \\ Italy; ${ }^{2}$ Philipps University of Marburg, \\ Marburg, Germany
}

Background: In order to reduce the risk of recurrence, adjuvant treatment with an aromatase inhibitor (AI) is recommended for postmenopausal women following surgery for hormone receptor-positive breast cancer. AIs are associated with improved disease-free survival compared with tamoxifen. The adverse events associated with AIs resemble those of menopause, such as bone density loss and musculoskeletal symptoms.

Purpose: We examine the case of a postmenopausal woman who was prescribed anastrozole, a nonsteroidal AI, as adjuvant therapy following surgery for estrogen and progesterone receptorpositive (ER and $\mathrm{PgR}+$ ) breast cancer.

Methods and sample: A 58-year-old postmenopausal woman diagnosed with ER and PgR+ breast cancer was prescribed anastrozole as adjuvant therapy following a right-inferior quadrantectomy. After experiencing joint pain and stiffness, she was prescribed paracetamol and a topical nonsteroidal anti-inflammatory drug. She was also counseled on nonpharmacological interventions. However, she continued to experience symptoms, and reported that she was not taking anastrozole regularly.

Results: The case study patient ultimately found relief by switching to letrozole, another aromatase inhibitor. This approach is supported by recent studies examining the benefits of switching strategies between aromatase inhibitors in order to relieve symptoms of arthralgia/myalgia.

Conclusions: Both adherence and strategies for managing aromatase inhibitor-associated arthralgia are key to deriving maximal clinical benefit from AI therapy. Switching from one aromatase inhibitor to another may provide a viable option in managing adverse events and enhancing adherence to medication.

Keywords: adherence, anastrozole, aromatase inhibitor, arthralgia, breast cancer, letrozole

\section{Background}

Guidelines such as the 2011 St Gallen International Consensus Statement, the 2010 European Society for Medical Oncology (ESMO), and American Society of Clinical Oncology (ASCO) guidelines, and the 2011 National Comprehensive Cancer Network (NCCN) guidelines include recommendations that, following surgery for early hormone receptor-positive $\left(\mathrm{HR}^{+}\right)$breast cancer, postmenopausal women receive adjuvant aromatase inhibitor (AI) treatment either as initial therapy or following 2-3 years of tamoxifen in order to reduce the risk of recurrence. ${ }^{1-4}$ The use of AIs as initial therapy, after 2-3 years of tamoxifen or after 5 years of tamoxifen, has been shown to be more effective than 5 years of adjuvant tamoxifen alone, resulting in an improvement in disease-free survival (DFS) and a reduction in breast cancer events. ${ }^{3,5}$ The ASCO recommendations take into account the fact that locoregional, distant recurrent, and
Correspondence: Jane Bryce National Cancer Institute, Via Mariano Semmola, 80I3I Napoli, Italy $\mathrm{Tel}+3908 \mathrm{I} 590357 \mathrm{I}$ ext 448 Fax +390817702938

Email jane.bryce@usc-intnapoli.net 
contralateral breast cancer have clinical consequences for breast cancer patients. ${ }^{3}$ The course of the disease is characterized by peaks in recurrence at 2 years and at 3.5 to 4 years following surgery for breast cancer; the majority of these recurrences are distant recurrences. ${ }^{6}$

In the Breast International Group (BIG) 1-98 trial, for example, upfront letrozole demonstrated a significant $19 \%$ improvement in DFS (351 events with letrozole vs 428 events with tamoxifen; $P=0.003$ ), and a significant $27 \%$ reduction in the risk of distant recurrence early on at 25.8 months of follow-up (184 events with letrozole vs 249 with tamoxifen; $P=0.001) .{ }^{7}$ The early reduction in the risk of distant metastases with letrozole seemed to have had longterm clinical implications on patient outcomes. At 76 months' median follow-up in BIG 1-98, there was a significant $12 \%$ improvement in DFS (509 events vs 565 events; $P=0.03$ ) and also a nonsignificant $13 \%$ improvement in overall survival $(P=0.08)$ with initial adjuvant letrozole compared with tamoxifen. ${ }^{8}$ Recently published data from the BIG 1-98 trial at 8 years' follow-up demonstrated a significant advantage in overall survival with letrozole monotherapy over tamoxifen monotherapy. ${ }^{9}$

Although the recommended duration of adjuvant AI therapy currently is 5 years according to the ESMO guidelines, ${ }^{2}$ the NCCN guidelines note that the optimal duration of AI therapy is not known, ${ }^{4}$ and trials are currently investigating durations of up to 10 years. ${ }^{10-12}$ It is important to communicate with patients about how to prevent and manage the adverse events associated with AIs, which resemble the symptoms of menopause and include bone density loss, increased fractures, arthralgia, and other musculoskeletal symptoms, ${ }^{3,13}$ and to reinforce the importance of staying on therapy to derive clinical benefits.

\section{Case presentation}

A 58-year-old postmenopausal woman had undergone natural menopause at 52 years old. She had no previous surgery prior to being diagnosed with breast cancer. Her body mass index (BMI) was $24.5 \mathrm{~kg} / \mathrm{m}^{2}$ (height $=169 \mathrm{~cm}$; weight $=70 \mathrm{~kg}$ ). She did housework and considered herself active. She had a history of mild irritable bowel syndrome (IBS) triggered by certain foods and by stress. She had been taking calcium (1000 mg) and vitamin D (400 international units) as directed by her family physician, since the onset of menopause.

In 2007, a mass in her right breast was discovered by routine mammography. In 2007, the patient had a right inferior quadrantectomy with sentinel lymph node (SLN) biopsy for breast cancer, and a 1-cm tumor and two SLNs were resected.
Pathology revealed that the tumor had tubular histology and was HER2 negative, and estrogen and progesterone receptor (ER and $\mathrm{PgR}$ ) positive; both SLNs were negative for metastases. In July through September 2007, the patient underwent radiation therapy to the right breast. Scintigraphy for bone metastases, X-ray for lung and liver metastases, and ultrasound for liver metastasis were all performed, and each was negative. Her medical oncologist recommended 5 years of adjuvant endocrine therapy with an AI, and in July 2007 , the patient initiated treatment with anastrozole $1 \mathrm{mg} /$ day. A postoperative bone mineral density test showed borderline osteopenia (T-scores: lumbar spine -1.5; left femoral -0.9; right femoral -1.3 ), and the patient continued previous calcium and vitamin D supplementation.

The patient was seen in the clinic for follow-up every 3 months for the next 24 months. At the first visit, she had complaints of joint pain and stiffness, particularly in her wrists and knees, but she reported no effect on her normal activities. She did not want to take any medication for the stiffness, and she reported taking anastrozole $1 \mathrm{mg}$ /day regularly. Paracetamol $1000 \mathrm{mg}$ was prescribed on an as-needed basis with a follow-up phone call planned after 2 weeks.

At the follow-up phone call, the patient reported no relief with paracetamol, but she did not want to take additional medications. Nonsteroidal anti-inflammatory drugs (NSAIDs) were suggested, but the patient had experienced problems in the past with this type of medication aggravating her intestinal symptoms. The problem of joint pain associated with AIs was reviewed with the patient, and nonpharmacological treatments such as stretching exercises, massage, and warm showers were suggested. The patient was not taking any homeopathic agents. The patient was still being seen every 3 months, and an evaluation was planned at 6 months.

At her 6-month visit, the patient reported being tired and achy, and she questioned how long AI therapy needed to continue. She reported taking anastrozole $1 \mathrm{mg}$ /day regularly and paracetamol occasionally (1000 mg, 2-3 times per day), but was not doing any specific exercises, yoga, or physical therapy. The patient was prescribed a topical NSAID: diclofenac gel 1\%, applied 2-3 times per day to affected areas. This led to some reduction in pain, and stiffness subsided, but the patient did not persist in using the topical formula as it was not effective in managing her pain.

At her 9-month visit, the patient was finding it difficult to keep up with her daily activities. She tried hot water baths at a nearby thermal spa, but did not persist with this intervention. The patient admitted that she had not been 
taking anastrozole $1 \mathrm{mg} /$ day regularly for the past month due to adverse event experiences. At this point, the option of a treatment change was discussed; it was decided that she would try a different AI to see if it might be tolerated better, and letrozole $2.5 \mathrm{mg}$ /day was then prescribed. The patient was to begin therapy 2 weeks after the visit, and have a clinic appointment after another 2 weeks.

The patient noticed an improvement in her joint symptoms after stopping anastrozole treatment. At the 2-week visit, she reported taking letrozole daily and was not experiencing joint stiffness. She received phone follow-ups at 2-week intervals, and at her clinic visit in July 2008, was no longer suffering from joint pain or stiffness. The patient is now 3 years past her initial breast cancer surgery and has been taking letrozole $2.5 \mathrm{mg}$ /day for more than 2 years.

\section{Discussion}

\section{Adjuvant Al clinical trials: incidence of musculoskeletal symptoms}

In clinical trials in postmenopausal women with $\mathrm{HR}^{+}$breast cancer, both nonsteroidal (anastrozole and letrozole) and steroidal (exemestane) AIs have been associated with an increased incidence of arthralgia compared with tamoxifen or placebo. In the anastrozole (Arimidex; AstraZeneca, Wilmington, DE), tamoxifen, alone or in combination (ATAC) trial of 5 years of initial adjuvant anastrozole compared with tamoxifen, the incidence of arthralgia at 68 months' follow-up was significantly higher with anastrozole than with tamoxifen $(35.6 \%$ vs $29.4 \% ; P<0.0001) .{ }^{14}$ It was noted that adverse events such as arthralgia were only recorded during the 5 years of active treatment and within the first 2 weeks after stopping treatment. ${ }^{15}$ Consequently, at 100 months of follow-up in ATAC, there was little difference in the incidence of arthralgia compared with previous reports, ${ }^{15}$ and the incidence of arthralgia was not reported at 120 months of follow-up. ${ }^{16}$ In the BIG 1-98 trial, at 71 months of median follow-up, arthralgia was more common in women taking letrozole-containing regimens (letrozole monotherapy for 5 years, or the sequence of 2 years of letrozole or tamoxifen followed by 3 years of the other agent) than in women receiving tamoxifen monotherapy for 5 years $(31.9 \%-34.7 \%$ vs $30.1 \% ; P=0.05) .{ }^{8}$ In the tamoxifen exemestane adjuvant multinational (TEAM) trial, at 2.75 years' median follow-up, the incidence of arthralgia was $17.9 \%$ with initial adjuvant exemestane and $9.2 \%$ with initial adjuvant tamoxifen $(P \leq 0.001) ;{ }^{17}$ at 5.1 years of median follow-up, the incidence of joint disorders was $36 \%$ with exemestane and $31 \%$ with sequential tamoxifen followed by exemestane $(P<0.0001) .{ }^{18}$ Similar results were seen in the Intergroup Exemestane Study (IES) trial, in which patients were randomized after completing 2-3 years of tamoxifen. ${ }^{19}$ At 55.7 months of median follow-up, significantly more patients who switched to exemestane reported arthralgia compared with patients who continued on tamoxifen for the rest of the 5-year treatment period (18.6\% vs $11.8 \%$; $P<0.0001) .{ }^{19}$ In an analysis at 91 months of median follow-up, including patients both on and post-treatment, the incidence of musculoskeletal pain was significantly higher with exemestane than with tamoxifen $(37.6 \%$ vs $29.9 \%$; $P<0.001) .{ }^{20}$ In the MA.17 trial of extended adjuvant letrozole compared with placebo in patients who had completed 5 years of tamoxifen treatment, there was significantly more arthralgia at 30 months of median follow-up with letrozole than with placebo $(25 \%$ vs $21 \% ; P<0.001){ }^{21}$

\section{Adherence to adjuvant $\mathrm{Al}$ therapy}

Suboptimal adherence to adjuvant medication regimen in postmenopausal women with $\mathrm{HR}^{+}$breast cancer may lead to inappropriate therapeutic efficacy of prescribed medication (tamoxifen or an AI), and may also increase the risk of cancer recurrence and reduce disease survival rates. Recently published reviews indicate that nonadherence with tamoxifen and AI therapy is common, and adherence reported in clinical trials may differ significantly from that observed outside of the clinical trial setting. ${ }^{22,23}$ Studies of patients taking adjuvant endocrine therapy for $\mathrm{HR}^{+}$breast cancer have shown nonadherence rates of $20 \%-50 \%$ at follow-up times ranging from $1-4.5$ years following the start of endocrine therapy. ${ }^{24-27}$

Joint symptoms are frequently cited as reasons for discontinuing treatment. ${ }^{27-29}$ In a retrospective study of 600 postmenopausal, $\mathrm{HR}^{+}$, early breast cancer patients receiving adjuvant AI therapy in a clinical practice setting (median follow-up 19.7 months), arthralgia and myalgia led to discontinuation of the $\mathrm{AI}$ in $45.5 \%$ and $17.8 \%$ of the 101 patients who discontinued due to toxicity, respectively. ${ }^{28}$ In another study, $20 \%$ of 56 breast cancer patients receiving AI therapy in a clinical practice setting discontinued due to new or worsening arthralgia or bone pain. ${ }^{29}$

Communicating with patients about AIs and presenting management strategies can help patients remain on treatment. ${ }^{27}$ The previously mentioned study of 56 patients in a clinical practice setting showed that discontinuation due to arthralgia or bone pain occurred within the first 3 months of treatment, leading the authors to recommend monthly visits during the first 3 months of therapy in order 
to address any issues and ensure that patients continue taking AIs. ${ }^{29}$ However, monthly visits are generally not an option for patients in Europe and North America, and were not an option for the patient in our case.

It is also important to communicate with patients about the benefits associated with AIs and the risks of not taking their prescribed treatment. ${ }^{27}$ The use of adjuvant AIs has been associated with significant improvements in DFS in postmenopausal women with $\mathrm{HR}^{+}$breast cancer (Table 1). A study using automated pharmacy records in a large integrated health system showed an increased risk of mortality at 10 years in women with Stage I-III HR+ breast cancer who discontinued or were nonadherent to endocrine therapy; survival was significantly higher in patients who adhered to therapy compared with patients who were nonadherent $(81.7 \%$ vs $77.8 \% ; P<0.01) .{ }^{24}$ A study of breast cancer patients in a community setting showed that 1589 (35\%) of the 4526 patients receiving endocrine therapy had adherence rates that have been associated with an increased risk of mortality (adherence rates $<80 \%$ ). ${ }^{30}$

Nurses can play a vital role in discussing adverse events with patients at each visit and also discerning whether they are taking their medications as prescribed. It has been suggested that follow-up phone calls between visits may also be helpful in addressing any adverse events that may arise. ${ }^{27}$ Other techniques to improve adherence include the use of aids such as calendars or pillboxes. ${ }^{27}$ Enlisting support from the patient's family and friends is another important component to facilitating patient adherence. ${ }^{27}$

\section{Managing musculoskeletal symptoms}

Recommendations for the management of joint symptoms include pharmacological and nonpharmacological interventions, and also switching to a different AI. Breast cancer patients taking AIs have used NSAIDs such as ibuprofen as well as paracetamol for the relief of joint symptoms. ${ }^{29}$ The patient in our case was prescribed paracetamol, which provided little relief. She did not want to take NSAIDs because of intestinal issues. However, it should be kept in mind that proton-pump inhibitors may be helpful in alleviating any gastrointestinal symptoms associated with NSAIDs. ${ }^{31}$ Use of a topical NSAID was associated with some relief of the patient's pain, but not stiffness, and she did not continue using it.

As an alternative to painkillers, a number of different nutritional supplements are being investigated for the treatment of arthralgia associated with the use of AIs. A Phase II clinical study evaluating glucosamine plus chondroitin (estimated enrollment $=53$ ) for the relief of joint pain and stiffness in postmenopausal breast cancer patients taking AIs is currently recruiting participants. ${ }^{32}$ In a report from this study that included 21 evaluable patients at 24 weeks, $80 \%$ of patients had experienced improvements in joint symptoms in their hands, knees, or both areas. ${ }^{33}$ Supplementation with vitamin D to achieve a target concentration of $40 \mathrm{ng} / \mathrm{mL} 25$-hydroxyvitamin $\mathrm{D}$ has also been associated with a decreased risk of new and worsening arthralgia in a prospective cohort study of postmenopausal breast cancer patients initiating AI therapy. ${ }^{34}$ Additional studies (one recently completed Phase I/II study and two Phase II studies currently in progress) have been investigating the use of vitamin D for arthralgia in postmenopausal women with breast cancer who are taking AIs. ${ }^{35-37}$

Nonpharmacological treatments for arthralgia associated with AIs include lifestyle changes such as exercise and weight loss; these may be more appropriate for patients with mild symptoms. ${ }^{31}$ Interestingly, compared with postmenopausal breast cancer patients with $\mathrm{BMI}<25 \mathrm{~kg} / \mathrm{m}^{2}$, the prevalence

Table I DFS benefits in selected trials of adjuvant Al therapy in postmenopausal women with $\mathrm{HR}^{+}$breast cancer $7,8,14,16-21,45$

\begin{tabular}{|c|c|c|c|c|}
\hline Clinical trial & Follow-up & Treatment arms & Adjuvant setting & DFS \\
\hline ATAC & 68 months & Anastrozole vs tamoxifen & Initial & $\mathrm{HR}=0.83 ; P=0.005^{\mathrm{a}}$ \\
\hline ATAC & 100 months & Anastrozole vs tamoxifen & Initial & $\mathrm{HR}=0.85 ; P=0.003^{\mathrm{a}}$ \\
\hline ATAC & 120 months & Anastrozole vs tamoxifen & Initial & $\mathrm{HR}=0.86 ; P=0.003^{\mathrm{a}}$ \\
\hline BIG I-98 & 25.8 months & Letrozole vs tamoxifen & Initial & $\mathrm{HR}=0.81 ; P=0.003$ \\
\hline BIG I-98 & 76 months & Letrozole vs tamoxifen & Initial & $\mathrm{HR}=0.88 ; P=0.03$ \\
\hline TEAM & 2.75 years & Exemestane vs tamoxifen & Initial & $\mathrm{HR}=0.89 ; P=0.12$ \\
\hline TEAM & 5.1 years & Exemestane vs tamoxifen $\rightarrow$ exemestane & Sequential & $\mathrm{HR}=0.97 ; P=0.60^{\mathrm{b}}$ \\
\hline IES & 55.7 months & Exemestane vs tamoxifen & Switch & $\mathrm{HR}=0.76 ; P=0.000 \mathrm{I}$ \\
\hline IES & 91 months & Exemestane vs tamoxifen & Switch & $\mathrm{HR}=0.84 ; P=0.002$ \\
\hline MA. 17 & 30 months & Letrozole vs placebo & Extended & $\mathrm{HR}=0.58 ; P<0.00 \mathrm{I}$ \\
\hline
\end{tabular}

Notes: ${ }^{a}$ Results for $\mathrm{HR}^{+}$subpopulation; ${ }^{\circ} 5$-year DFS.

Abbreviations: Al, aromatase inhibitor; ATAC, anastrozole (Arimidex), tamoxifen, alone or in combination; BIG, Breast International Group; DFS, disease-free survival; $\mathrm{HR}^{+}$, hormone receptor-positive; IES, Intergroup Exemestane Study; TEAM, tamoxifen exemestane adjuvant multinational. 
Table 2 Studies of switching from one Al to another in postmenopausal women with $\mathrm{HR}^{+}$breast cancer to address musculoskeletal symptoms

\begin{tabular}{|c|c|c|c|c|}
\hline Study & $\mathbf{N}$ & Study design & Results & Reference \\
\hline ALIQUOT & 182 & $\begin{array}{l}\text { Open-label, crossover study } \\
12 \text { weeks of letrozole followed by } 12 \text { weeks of anastrozole, } \\
\text { or } 12 \text { weeks of anastrozole followed by } 12 \text { weeks of letrozole }\end{array}$ & $\begin{array}{l}56 \% \text { had joint problems on letrozole } \\
\text { but not on anastrozole } \\
55 \% \text { had joint problems on anastrozole } \\
\text { but not on letrozole }\end{array}$ & $4 I, 42$ \\
\hline ATOLL & 179 & $\begin{array}{l}\text { Prospective, nonrandomized, open-label, multicenter study } \\
\text { Patients who discontinued anastrozole due to musculoskeletal } \\
\text { symptoms initiated letrozole for } 6 \text { months or until } \\
\text { discontinuation due to intolerance or disease progression }\end{array}$ & $\begin{array}{l}71.5 \% \text { of patients on letrozole at } \\
6 \text { months } \\
28.5 \% \text { discontinued due to } \\
\text { musculoskeletal symptoms }\end{array}$ & 43 \\
\hline Yardley et al ${ }^{44}$ & 261 & $\begin{array}{l}\text { Patients who discontinued anastrozole due to grade } 2 \text { or } 3 \\
\text { arthralgia/myalgia initiated letrozole for } 6 \text { months }\end{array}$ & $\begin{array}{l}87.4 \% \text { of patients on letrozole at } \\
6 \text { months } \\
9.6 \% \text { discontinued due to arthralgia/ } \\
\text { myalgia }\end{array}$ & 44 \\
\hline
\end{tabular}

Abbreviations: Al, aromatase inhibitor; ALIQUOT, Anastrozole versus Letrozole: Investigation into Quality of Life and Tolerability; ATOLL, Articular Tolerance of Letrozole; $\mathrm{HR}^{+}$, hormone receptor-positive.

of joint pain appears to be reduced in postmenopausal breast cancer patients who are overweight (BMI $25-30 \mathrm{~kg} / \mathrm{m}^{2}$ ) but not in those who are obese $\left(\mathrm{BMI}>30 \mathrm{~kg} / \mathrm{m}^{2}\right) .{ }^{38}$ This observation may be attributed to increased adipose tissue in overweight women, resulting in increased levels of estrogen and other sex hormones, since joint problems in women taking AIs or in normal menopause appear to be related to a reduction in estrogen levels. ${ }^{38}$ However, obesity is associated with an increased risk of osteoarthritis; this may negate the protection from arthralgia afforded by the increased levels of estrogen. ${ }^{38}$

Warm showers, physical therapy, massage, and acupuncture may also provide relief of arthralgia symptoms. ${ }^{39,40}$ In a randomized, blinded, sham-controlled study in 51 postmenopausal patients with early breast cancer and self-reported musculoskeletal pain while taking AIs, acupuncture was effective in reducing joint pain and stiffness and improving functional ability and physical wellbeing. ${ }^{40}$ Several of these interventions were presented to our patient, but she was not amenable to physical therapy or exercises, and did not persist in going to a thermal spa for the temporary relief she received from hot water baths.

Switching from one nonsteroidal AI to another is another strategy that may allow patients experiencing arthralgia on one AI to continue adjuvant AI therapy. This is the treatment strategy that ultimately allowed our patient to find relief for her symptoms and to continue the benefits of adjuvant AI therapy. In the Anastrozole versus Letrozole: Investigation into Quality of Life and Tolerability (ALIQUOT) study of adjuvant AIs in postmenopausal women with $\mathrm{HR}^{+}$breast cancer, the incidence of joint pain was similar with letrozole and with anastrozole $(40 \%-49 \%$ vs $52 \%) .{ }^{41}$ However, approximately half of the patients in ALIQUOT with joint symptoms on letrozole did not report joint symptoms on anastrozole, and vice versa (Table 2) ${ }^{42}$ In the Articular Tolerance of Letrozole (ATOLL) study and a study by Yardley et al, a majority of postmenopausal women with $\mathrm{HR}^{+}$breast cancer who discontinued anastrozole due to musculoskeletal symptoms were able to continue therapy with letrozole for at least 6 months: $71.5 \%$ in ATOLL and 87.4\% in Yardley et al (Table 2). ${ }^{43,44}$

\section{Conclusions}

Approaches to alleviate musculoskeletal symptoms in postmenopausal breast cancer patients taking AIs include pharmacological interventions (such as NSAIDs or vitamin D supplementation), nonpharmacological interventions (such as homeopathy or lifestyle changes including exercise and weight loss), and switching between nonsteroidal AIs. While offering greater clinical efficacy than tamoxifen, switching from one AI to another can provide relief of joint pain and stiffness for some postmenopausal women taking adjuvant AIs for $\mathrm{HR}^{+}$breast cancer. It is important to address the adverse events associated with AIs and also to provide strategies for managing them so that in order to derive the most clinical benefit, patients remain adherent to therapy.

\section{Disclosures}

Financial support for medical editorial assistance was provided by Novartis Pharmaceuticals, East Hanover, NJ. $\mathrm{PH}$ reports past consultancy, grant support, and lecture fees from AstraZeneca, Novartis, Pfizer Inc, Roche, Eli Lilly, and Amgen. MB and JB report no conflicts of interest. The authors thank Dr Maria Soushko of Phase Five Communications Inc for her medical editorial assistance with this manuscript. 


\section{References}

1. Gnant M, Harbeck N, Thomssen C. St Gallen 2011: Summary of the Consensus Discussion. Breast Care (Basel). 2011;6(2):136-141.

2. Aebi S, Davidson T, Gruber G, Castiglione M, ESMO Guidelines Working Group. Primary breast cancer: ESMO Clinical Practice Guidelines for diagnosis, treatment and follow-up. Ann Oncol. 2010; 21(Suppl 5):v9-v14.

3. Burstein HJ, Prestrud AA, Seidenfeld J, et al; American Society of Clinical Oncology. American Society of Clinical Oncology clinical practice guideline: update on adjuvant endocrine therapy for women with hormone receptor-positive breast cancer. J Clin Oncol. 2010;28:3784-3796.

4. National Comprehensive Cancer Network (NCCN). NCCN Clinical Practice Guidelines in Oncology. Breast Cancer. V2.2011. Available from: http://www.nccn.org/professionals/physician_gls/f_guidelines. asp. Accessed May 1, 2011.

5. Goldhirsch A, Ingle JN, Gelber RD, et al; Panel members. Thresholds for therapies: highlights of the St Gallen International Expert Consensus on the primary therapy of early breast cancer 2009. Ann Oncol. 2009;20(8):1319-1329.

6. Mansell J, Monypenny IJ, Skene AI, et al. Patterns and predictors of early recurrence in postmenopausal women with estrogen receptorpositive early breast cancer. Breast Cancer Res Treat. 2009;117(1): 91-98.

7. Breast International Group (BIG) 1-98 Collaborative Group, Thürlimann B, Keshaviah A, et al. A comparison of letrozole and tamoxifen in postmenopausal women with early breast cancer. $N$ Engl J Med. 2005;353(26):2747-2757.

8. BIG 1-98 Collaborative Group, Mouridsen H, Giobbie-Hurder A, et al. Letrozole therapy alone or in sequence with tamoxifen in women with breast cancer. N Engl J Med. 2009;361(8):766-776.

9. Regan MM, Neven P, Giobbie-Hurder A, et al. Assessment of letrozole and tamoxifen alone and in sequence for postmenopausal women with steroid hormone receptor-positive breast cancer: the BIG 1-98 randomised clinical trial at $8 \cdot 1$ years median follow-up. Lancet Oncol. 2011;12(12):1101-1108.

10. AstraZeneca. Secondary adjuvant long term study with arimidex (SALSA). In: ClinicalTrials.gov [website on the Internet]. Bethesda, MD: US National Library of Medicine; 2000. Available from: http://www.clinicaltrials.gov/ct2/show/NCT00295620. NLM Identifier: NCT00295620. Accessed May 1, 2011.

11. National Surgical Adjuvant Breast and Bowel Project (NSABP); National Cancer Institute (NCI); Novartis. Letrozole in treating postmenopausal women who have received hormone therapy for hormone receptor-positive breast cancer. In: ClinicalTrials.gov [website on the Internet]. Bethesda, MD: US National Library of Medicine; 2000. Available from: http://www.clinicaltrials.gov/ct2/ show/NCT00382070. NLM Identifier: NCT00382070. Accessed May 1, 2011.

12. International Breast Cancer Study Group; National Cancer Institute (NCI). Letrozole in preventing cancer in postmenopausal women who have received 4-6 years of hormone therapy for hormone receptor-positive, lymph node-positive, early-stage breast cancer. In: ClinicalTrials.gov [website on Internet]. Bethesda, MD: US National Library of Medicine; 2000. Available from: http://www.clinicaltrials.gov/ct2/ show/NCT00553410. NLM Identifier: NCT00553410. Accessed May 1, 2011.

13. Hadji P. Menopausal symptoms and adjuvant therapy-associated adverse events. Endocr Relat Cancer. 2008;15(1):73-90.

14. Howell A, Cuzick J, Baum M, et al; ATAC Trialists' Group. Results of the ATAC (Arimidex, Tamoxifen, Alone or in Combination) trial after completion of 5 years' adjuvant treatment for breast cancer. Lancet. 2005;365(9453):60-62.

15. Arimidex, Tamoxifen, Alone or in Combination (ATAC) Trialists' Group, Forbes JF, Cuzick J, et al. Effect of anastrozole and tamoxifen as adjuvant treatment for early-stage breast cancer: 100-month analysis of the ATAC trial. Lancet Oncol. 2008;9(1):45-53.
16. Cuzick J, Sestak I, Baum M, et al; ATAC/LATTE investigators. Effect of anastrozole and tamoxifen as adjuvant treatment for early-stage breast cancer: 10-year analysis of the ATAC trial. Lancet Oncol. 2010;11(12):1135-1141.

17. Jones SE, Seynaeve C, Hasenburg A, et al. Results of the first planned analysis of the TEAM (tamoxifen exemestane adjuvant multinational) prospective randomized phase III trial in hormone sensitive postmenopausal early breast cancer. Cancer Res. 2009; 69(Suppl 2; Abstr 15):66s.

18. van de Velde CJ, Rea D, Seynaeve C, et al. Adjuvant tamoxifen and exemestane in early breast cancer (TEAM): a randomised phase 3 trial. Lancet. 2011;377(9762):321-331.

19. Coombes RC, Kilburn LS, Snowdon CF, et al; Intergroup Exemestane Study. Survival and safety of exemestane versus tamoxifen after 2-3 years' tamoxifen treatment (Intergroup Exemestane Study): a randomised controlled trial. Lancet. 2007;369(9561):559-570.

20. Coombes R, Kilburn L, Beare S, Snowdon C, Bliss J. Imperial College London, Oncology, London, United Kingdom; Institute of Cancer Research, ICR Clinical Trials and Statistics Unit (ICR-CTSU), Sutton, United Kingdom. Survival and safety post study treatment completion: an updated analysis of the Intergroup Exemestane Study (IES) - submitted on behalf of the IES Investigators. Eur J Cancer. 2009;7(Supp1 2):263, Abst O-5010.

21. Goss PE, Ingle JN, Martino S, et al. Randomized trial of letrozole following tamoxifen as extended adjuvant therapy in receptor-positive breast cancer: updated findings from NCIC CTG MA.17. J Natl Cancer Inst. 2005;97(17):1262-1271.

22. Banning M. Adherence to adjuvant therapy in post-menopausal breast cancer patients: a review. Eur J Cancer Care (Engl). 2012;21(1):10-19.

23. Gotay C, Dunn J. Adherence to long-term adjuvant hormonal therapy for breast cancer. Expert Rev Pharmacoecon Outcomes Res. 2011;11(6):709-715.

24. Hershman DL, Shao T, Kushi LH, et al. Early discontinuation and nonadherence to adjuvant hormonal therapy are associated with increased mortality in women with breast cancer. Breast Cancer Res Treat. 2011;126(2):529-537.

25. Ziller V, Kalder M, Albert US, et al. Adherence to adjuvant endocrine therapy in postmenopausal women with breast cancer. Ann Oncol. 2009;20(3):431-436.

26. Partridge AH, LaFountain A, Mayer E, Taylor BS, Winer E, Asnis-Alibozek A. Adherence to initial adjuvant anastrozole therapy among women with early-stage breast cancer. J Clin Oncol. 2008;26(4):556-562.

27. Hadji P. Improving compliance and persistence to adjuvant tamoxifen and aromatase inhibitor therapy. Crit Rev Oncol Hematol. 2010; 73(2):156-166.

28. Dent SF, Hopkins S, DiValentin T, Verreault J, Vandermeer L, Verma S; The Ottawa Hospital Regional Cancer Centre (TOHRCC), Ottawa, ON, Canada. Adjuvant aromatase inhibitors in early breast cancer toxicity and adherence. Important observations in clinical practice. Breast Cancer Res Treat. 2007;106(Suppl 1):S111.

29. Presant CA, Bosserman L, Young T, et al. Aromatase inhibitor-associated arthralgia and/or bone pain: frequency and characterization in non-clinical trial patients. Clin Breast Cancer. 2007;7(10):775-778.

30. Makubate B, McCowan C, Dewar JA, Thompson AM. Completion of 5-years adjuvant endocrine therapy in the community. 33rd Annual San Antonio Breast Cancer Symposium; December 8-12, 2010; San Antonio, TX, USA. Abst P5-11-11.

31. Coleman RE, Bolten WW, Lansdown M, et al. Aromatase inhibitorinduced arthralgia: clinical experience and treatment recommendations. Cancer Treat Rev. 2008;34(3):275-282.

32. Columbia University. Glucosamine and chondroitin for aromatase inhibitor induced joint symptoms in women with breast cancer. In: ClinicalTrials.gov [website on the Internet]. Bethesda, MD: US National Library of Medicine; 2000. Available from: http:/www.clinicaltrials.gov/ct2/show/NCT00691678. NLM Identifier: NCT00691678. Accessed May 1, 2011. 
33. Greenlee H, Crew KD, Shao T, et al. Phase II study of glucosamine with chondroitin on joint symptoms induced by aromatase inhibitors in breast cancer patients. 33rd Annual San Antonio Breast Cancer Symposium; December 8-12, 2010; San Antonio, TX, USA. Abst P2-13-02.

34. Prieto-Alhambra D, Javaid MK, Servitja S, et al. Vitamin D threshold to prevent aromatase inhibitor-induced arthralgia: a prospective cohort study. Breast Cancer Res Treat. 2011;125(3):869-878.

35. Stanford University; Department of Defense. Ph I/II of vitamin D on bone mineral density and markers of bone resorption. In: ClinicalTrials.gov [website on the Internet]. Bethesda, MD: US National Library of Medicine; 2000. Available from: http://www.clinicaltrials.gov/ct2/ show/NCT00904423. NLM Identifier: NCT00904423. Accessed May 1, 2011.

36. University of Kansas; Novartis Pharmaceuticals BTR Group. Vitamin D3 for aromatase inhibitor induced arthralgias (VITAL). In: ClinicalTrials.gov [website on the Internet]. Bethesda, MD: US National Library of Medicine; 2000. Available from: http://www.clinicaltrials.gov/ ct2/show/NCT00867217. NLM Identifier: NCT00867217. Accessed May 1, 2011.

37. Fred Hutchinson Cancer Research Center; National Cancer Institute (NCI). Vitamin D deficiency, muscle pain, joint pain, and joint stiffness in postmenopausal women receiving letrozole for stage I-III breast cancer. In: ClinicalTrials.gov [website on the Internet]. Bethesda, MD: US National Library of Medicine; 2000. Available from: http://www.clinicaltrials.gov/ct2/show/NCT00416715. NLM Identifier: NCT00416715. Accessed May 1, 2011.

38. Crew KD, Greenlee H, Capodice J, et al. Prevalence of joint symptoms in postmenopausal women taking aromatase inhibitors for early-stage breast cancer. J Clin Oncol. 2007;25(25):3877-3883.
39. Thorne C. Clinical management of arthralgia and bone health in women undergoing adjuvant aromatase inhibitor therapy. Curr Opin Oncol. 2007;19(Suppl 1):S19-S28.

40. Crew KD, Capodice JL, Greenlee H, et al. Randomized, blinded, sham-controlled trial of acupuncture for the management of aromatase inhibitor-associated joint symptoms in women with early-stage breast cancer. J Clin Oncol. 2010:28(7):1154-1160.

41. Dixon JM, Renshaw L, Langridge C, et al. Anastrozole and letrozole: an investigation and comparison of quality of life and tolerability. Breast Cancer Res Treat. 2011;125(3):741-749.

42. Renshaw L, McHugh M, Williams L, et al. Comparison of joint problems as reported by patients in a randomised adjuvant trial of anastrozole and letrozole. Breast Cancer Res Treat. 2007;106(Suppl 1):S108, Abst 2072.

43. Briot K, Tubiana-Hulin M, Bastit L, Kloos I, Roux C. Effect of a switch of aromatase inhibitors on musculoskeletal symptoms in postmenopausal women with hormone-receptor-positive breast cancer: the ATOLL (articular tolerance of letrozole) study. Breast Cancer Res Treat. 2010;120(1):127-134.

44. Yardley D, Green N, Papish S, et al. Rheumatologic evaluation of adjuvant letrozole in post-menopausal breast cancer patients discontinuing anastrozole due to grade 2-3 arthralgia or myalgia. Cancer Res. 2009;69(24 Suppl 3):Abst 805.

45. Arimidex, Tamoxifen, Alone or in Combination (ATAC) Trialists' Group, Forbes JF, Cuzick J, et al. Effect of anastrozole and tamoxifen as adjuvant treatment for early-stage breast cancer: 100-month analysis of the ATAC trial. Lancet Oncol. 2008;9(1):45-53.
Cancer Management and Research

\section{Publish your work in this journal}

Cancer Management and Research is an international, peer-reviewed open access journal focusing on cancer research and the optimal use of preventative and integrated treatment interventions to achieve improved outcomes, enhanced survival and quality of life for the cancer patient The journal welcomes original research, clinical \& epidemiological studies, reviews \& evaluations, guidelines, expert opinion \& commentary, case reports \& extended reports. The manuscript management system is completely online and includes a very quick and fair peerreview system, which is all easy to use. Visit http://www.dovepress.com/ testimonials.php to read real quotes from published authors. 